\title{
Image Guided Surgery for dental implantology: an in-vivo prospective study evaluate its accuracy
}

CLINICAL INNOVATIONS
Pellegrino Gerardo*, Taraschi Valerio+, Andrea Zacchino*, Agnese Ferri ${ }^{\star}$, Claudio Marchetti ${ }^{\star \star}$

* Oral of Maxillofacial Surgery Division, DIBINEM, University of Bologna

+ Chiversity of Technology - Sydney, School of Mathematical and Physical Science, Australia

\section{Background and Aim}

Nowadays many surgical procedures, such as oral-maxillofacial, orthopaedic and brain surgery use Image -Guided Surgery, which can be a helpful tool for the surgeon as complex anatomy cases occur. Nevertheless, the evaluation of the surgical anatomy and the simulation of the procedures still represent a problem, due to the difficult combination of a virtual image with the real structure. ImplaNav ${ }^{\top M}$ (BresMedical, Sydney, 2015), an advanced system manufactured for oral and maxillofacial surgery navigation, builds a three-dimensional preview of the patient's anatomy, giving the surgeon the tools to plan the procedures with accuracy before operating and guiding his hands while placing implants as previously planned.

The aim of this in-vivo study is to evaluate the accuracy of the ImplaNav ${ }^{\mathrm{TM}}$ system.

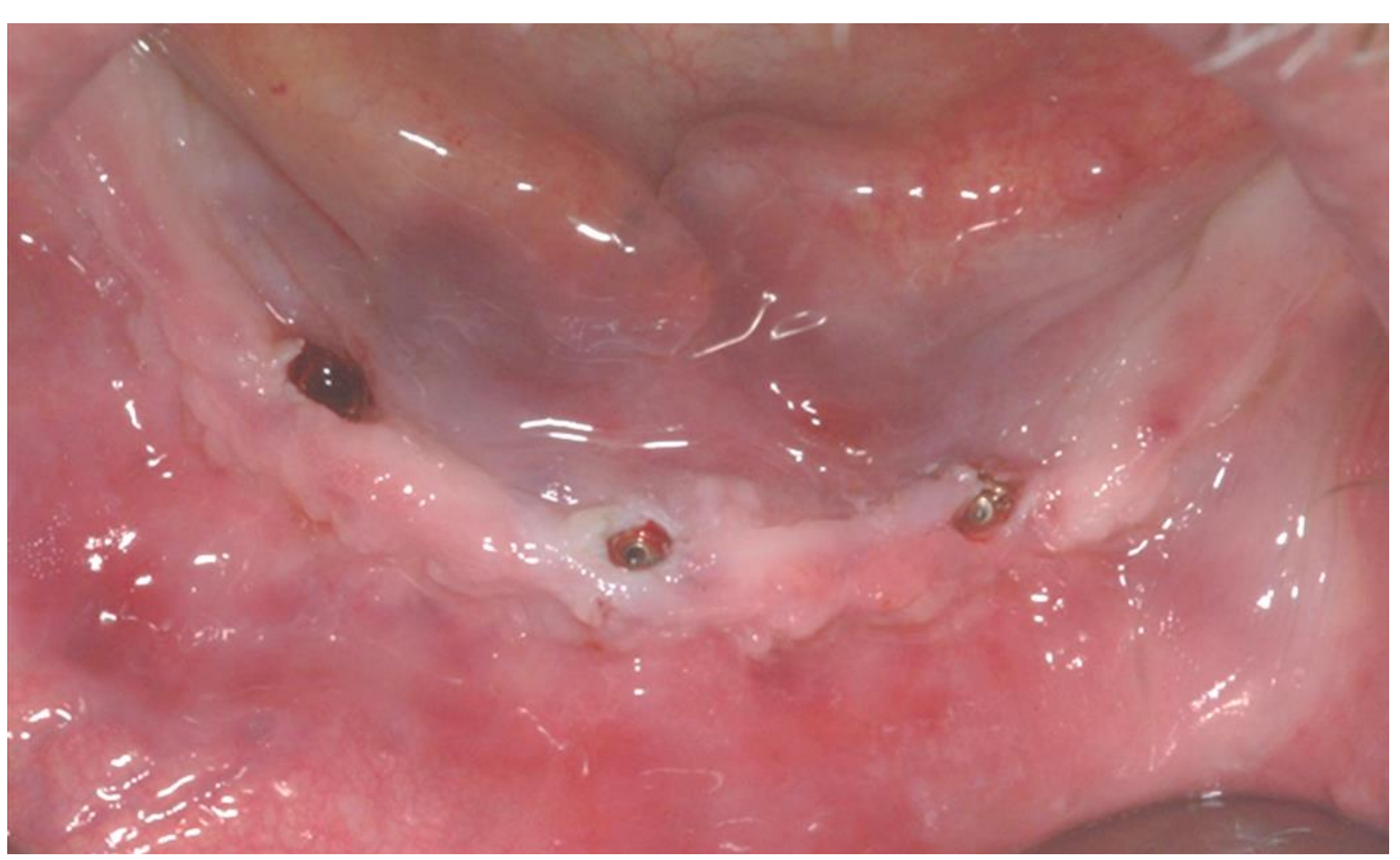

Fig. 1: Flapless implant placement with ImplaNav

\section{Methods and Materials}

Eight patients and ten implants were evaluated in these Clinical Trials approved by the Ethical Committee. Six patients were partially and two were totally edentulous. According to the navigation system protocol a teeth-supported or implant supported intra-oral reference tool was positioned before a Cone Beam CT scan. The ImplaNav ${ }^{\mathrm{TM}}$ software was used for the diagnosis and the virtual planning of maxillary and mandibular fixtures. The surgery was tracked by way of following the planning, in real-time, until the final implant position. A post-operative Cone Beam CT scan was undertaken within three months of the surgery The planned implants position and the real position of the implants obtained from the post-operative scan were aligned using a dedicated software for spatial registration. The deviation between the real position of the implants and the planned one was measured.

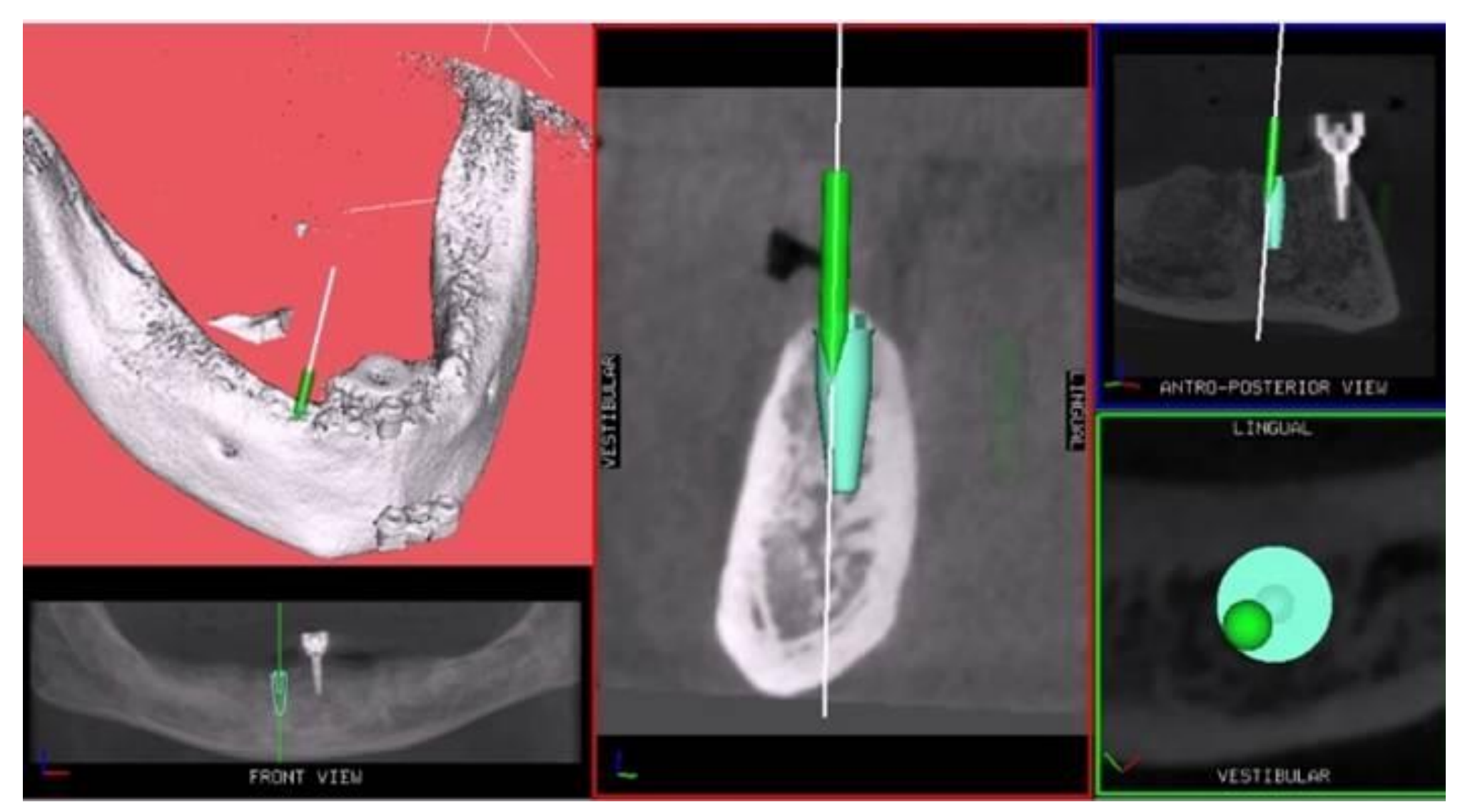

Fig. 2: The implant planning and the virtual position of the drill

\section{Results}

A maximum 3D spatial deviation between the inserted fixtures and the virtual ones of $0.77 \mathrm{~mm}$ was measured at the entry point reporting a standard deviation of $0.28 \mathrm{~mm}$. The deviation recorded at the apex was $0.94 \mathrm{~mm}$ with a standard deviation of $0.09 \mathrm{~mm}$. The mean angular deviation between the axes resulted in a 3.98 degrees angle.

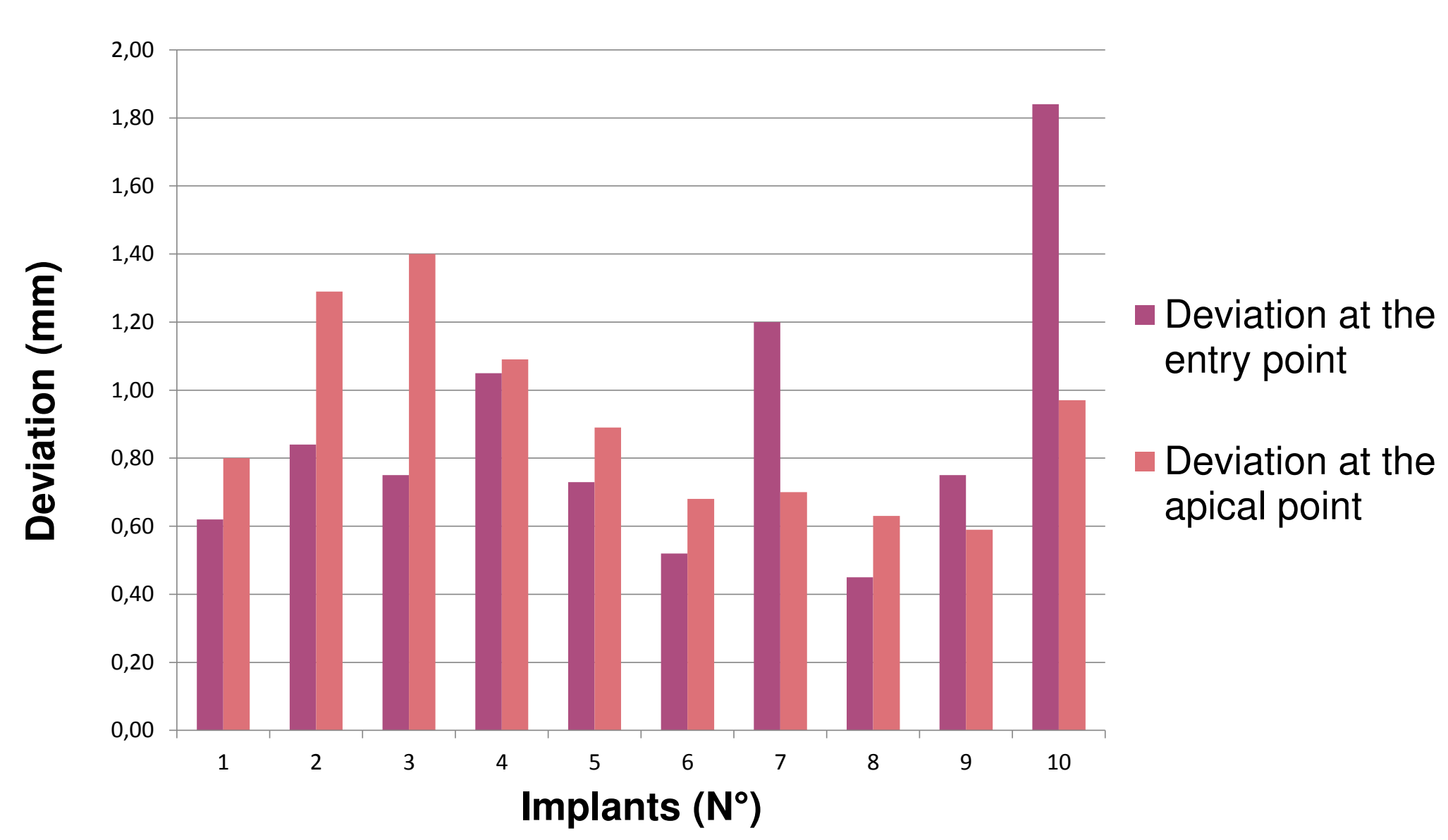

Fig. 3: Distribution of the deviations at the insertion point and at the apical point

\section{Conclusions}

The ImplaNav ${ }^{\top \mathrm{M}}$ system seems to be useful for the clinical practice. The accuracy reported in this study results comparable with the literature data found for dynamic navigation and drill guides systems. The simplified procedure does not result in additional costs for each treated patient and the possibility to work with a direct view of the operative field could introduce some significant advantages. The use of this system could be particularly suitable for advanced implantology like tilted or zygomatic implants. More clinical trials with a larger sample size are required to compare this system with others in the different clinical situations.

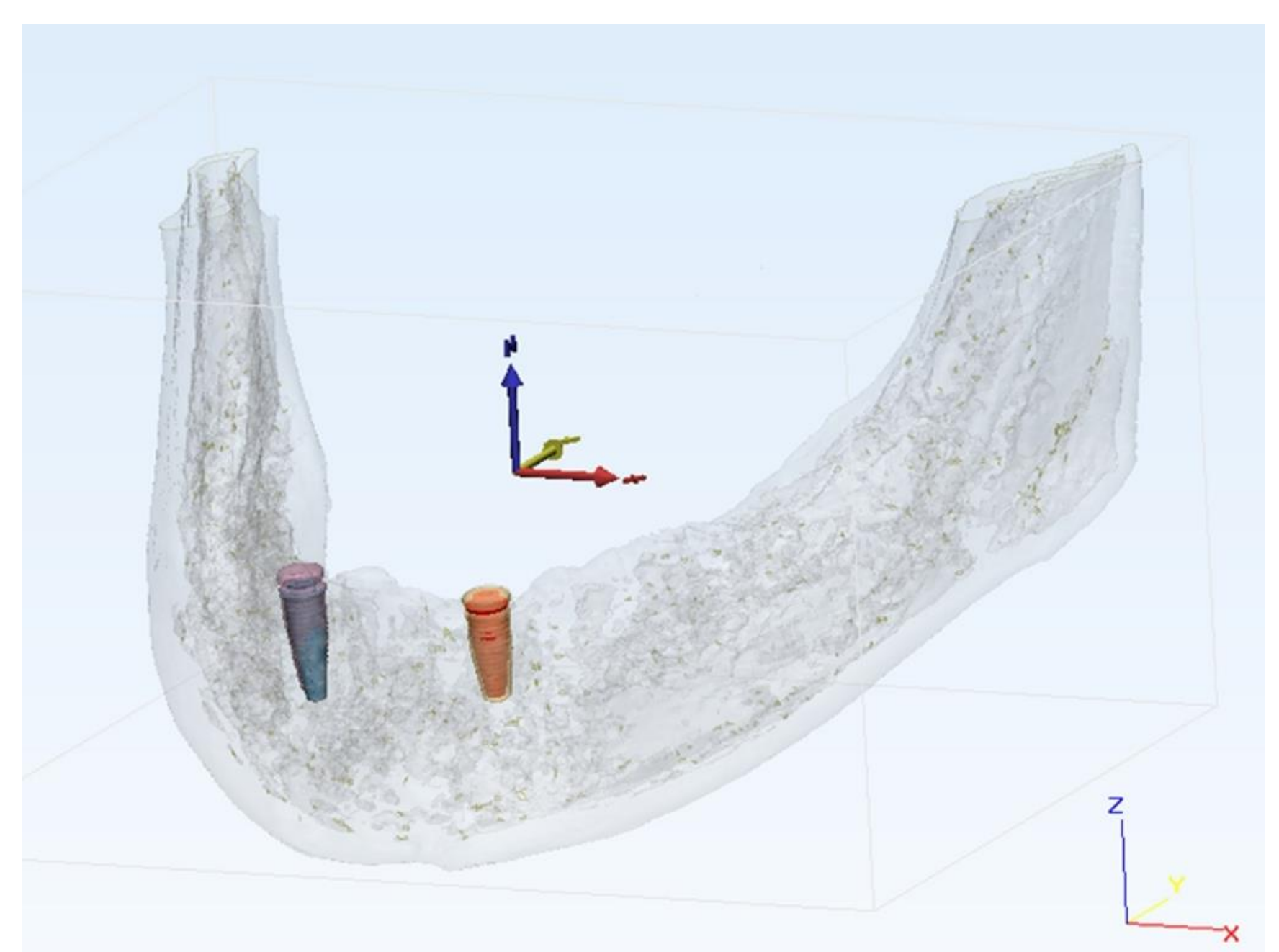

Fig. 4: Superimposition of the planned and the real position of implants

\section{References}

1. Block MS, Emery RW, Lank K, Ryan J. Implant Placement Accuracy Using Dynamic Navigation. Int J Oral Maxillofac Implants. 2017

2. Vercruyssen M, Fortin T, Widmann G, Jacobs R, Quirynen M. Different techniques of static/dynamic guided implant surgery: modalities and indications. Periodontol 2000. 2014

3. D'haese J, Ackhurst J, Wismeijer D, De Bruyn H, Tahmaseb A. Current state of the art of computer-guided implant surgery. Periodontol 2000. 2017 